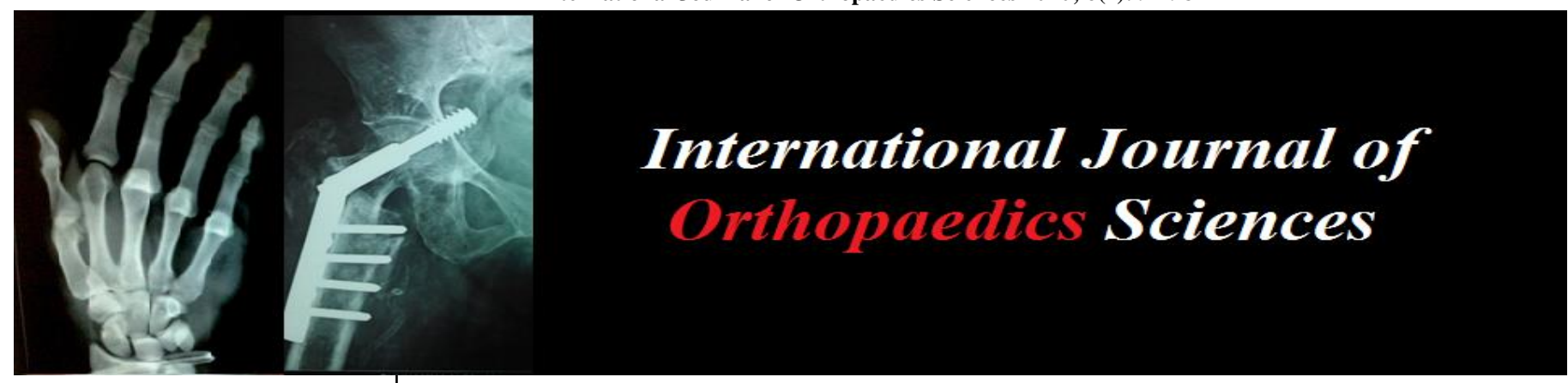

E-ISSN: 2395-1958

P-ISSN: 2706-6630

IJOS 2020; 6(2): 94-98

(C) 2020 IJOS

www.orthopaper.com

Received: 10-01-2020

Accepted: 13-02-2020

Dr. Vivekananda BR MBBS, MS Ortho, Assistant

Professor, Department of Orthopaedics, Sanjay Gandhi

Institute of Trauma and

Orthopaedics, Bengaluru,

Karnataka, India

Dr. Varun L Shetty

MBBS, MS Ortho, Senior

Resident, Department of

Orthopaedics, Sanjay Gandhi

Institute of Trauma and

Orthopaedics, Bengaluru,

Karnataka, India

Dr. Muthu Kishore M

MBBS, MS Ortho, Junior

Resident, Department of

Orthopaedics, Sanjay Gandhi

Institute of Trauma and

Orthopaedics, Bengaluru,

Karnataka, India

Dr. Sunilkumar TR

MBBS, MS Ortho, Assistant

Professor, Department of

Orthopaedics, Sanjay Gandhi

Institute of Trauma and

Orthopaedics, Bengaluru,

Karnataka, India

\section{Clinical outcome of open reduction and internal fixation of proximal humerus fractures with proximal humerus locking plates}

\author{
Dr. Vivekananda BR, Dr. Varun L Shetty, Dr. Muthu Kishore M and Dr. \\ Sunilkumar TR
}

DOI: https://doi.org/10.22271/ortho.2020.v6.i2b.2023

\begin{abstract}
Background and Objective: Proximal humerus fractures are one of the commonly encountered fractures in an orthopaedic setup comprising nearly $4 \%$ of all fractures. Achieving a stable fixation in three and four part fractures have always been a challenge. There is also a debate about the ideal method to treat such fractures. We undertook the present study to assess the functional outcome following proximal humerus fixation with proximal humerus locking plates.

Methods: This is a prospective study of 30 cases of proximal humerus fractures operated by ORIF with Proximal humerus locking plate from January 2018 to May 2019 at Sanjay Gandhi Institute of Trauma and Orthopaedics, Bengaluru. The cases were evaluated radiologically and functionally assessed using the Constant Murley score at the end of study.

Results: In our case series of patients, majority were males, with RTA being the commonest mode of injury in young population and domestic fall being the most common mode of injury in elderly. All the fractures united with a good to excellent outcome in $76 \%$ of the patients. Malunions were the only complications and there were no incidences of screw perforation, AVN, plate impingement and infection. Conclusion: In conclusion locking compression plate is an advantageous implant in proximal humeral fractures due to angular stability, particularly in comminuted osteoporotic bones in elderly patients, thus allowing early mobilization.
\end{abstract}

Keywords: Proximal humerus fracture, Constant Murley score

\section{Introduction}

Nearly $4 \%$ of all fractures and $26 \%$ of Humerus fractures are that of the Proximal Humerus ${ }^{[1]}$. The humeral head has an osseous architecture such that there is poor cancellous bone stock in the centre, which leads to high chances of failure with conventional plate and screw fixation. It has therefore been a challenge to achieve a stable fixation in three part or four part Proximal humerus fractures (constituting around $13-16 \%)^{[2]}$.

Various methods of fixations have been described, such as, Kirschner wires, External fixation, tension band wiring, rush pins and intramedullary nails ${ }^{[3]}$, but these are associated with many complications such as malunion, non union, avascular necrosis of the humeral head, rotator cuff dysfunction and joint stiffness ${ }^{[4]}$.

In order to improve screw fixation in osteoporotic bones and to minimize soft tissue dissection, the Proximal Humerus Locking Plate has been developed. The principles of fixation with a conventional plate have been combined with those of locking screws in this. It is contoured for the proximal Humerus being preshaped in this manner. There is no risk of loss of reduction and blood supply is preserved, as this requires no compression. Angular as well as axial stability is ensured by locking the screws into the plate. This also reduces the risk of loss of reduction. Subsidence in the metaphyseal area is prevented by the locked interface which also provides fixed stability ${ }^{[5]}$.

The patient would be unable to work and even carry out daily activities until the fracture heals and hence, fracture of the proximal humerus is a debilitating problem. Therefore, by evaluating the efficacy of the proximal humerus locking plates, we would be able to assess the functional outcome, pain, range of movements and ability to carry out the daily activities.
Corresponding Author: Dr. Varun L Shetty MBBS, MS Ortho, Senior Resident, Department of Orthopaedics, Sanjay Gandhi Institute of Trauma and Orthopaedics, Bengaluru, Karnataka, India 


\section{Materials and Methods}

Our study is a hospital based prospective study. The subjects for the study were the patients fulfilling the inclusion criteria admitted in Sanjay Gandhi Institute of Trauma and Orthopaedics, Bengaluru during the period from January 2018 to May 2019.

\section{Inclusion Criteria}

1. All skeletally mature patients presenting with displaced proximal Humerus fractures according to NEER two, three and four part fracture.

2. Open fractures of type 1,2 , and $3 a$

\section{Exclusion Criteria}

1. Patient refusal.

2. Pathologic fractures from primary or metastatic tumours

3. Patients age less than 18 years.

4. Patients having intra-articular fractures of ipsilateral elbow, ipsilateral clavicle fractures involving acromioclavicular joint, ipsilateral scapular fractures extending into glenohumeral joint.

5. Isolated greater and lesser tuberosity fractures.

6. Head splitting fractures.

7. Patient not fit for surgery due to medical problem.

\section{Steps of Data Collection}

A detailed history from patients and their attenders followed by a clinical examination was done, both local and systemic. Following this, they were assessed radiologically with X-rays
-AP and Axillary view along with CT scan with 3D reconstruction. Investigations required for fitness for surgery was done which included complete hemogram, blood grouping and Rh typing, Blood urea, Serum creatinine, Serum electrolytes, Random blood sugar, $\mathrm{Hbs} \mathrm{Ag} / \mathrm{HCV} / \mathrm{HIV}$ and ECG. The fractures were then classified according to Neer's classification.

Patients fit for surgery were operated with open reduction and internal fixation with proximal humerus locking plate under brachial block or general anesthesia. Position used for the procedure was either a Beach chair or supine, and the approach used was Deltopectoral approach. The fractures were reduced and held with $\mathrm{k}$ wires and reduction checked under fluoroscopy followed by final fixation by the locking plate.

Postoperatively arm pouch was used to immobilize the operated limb and pendulum exercises as per patient's tolerance were started on post operative day 1 .

Postoperative $\mathrm{x}$ rays were done in AP and axillary view to assess the reduction and stability of fracture fixation. Suture/staple removal was done on post-op day 10 and then patients discharged with arm pouch and adviced to continue pendulum exercises. Patients were followed up on OPD basis at intervals of 6 Weeks, 12 Weeks, and 6 Months. During this period, in each visit clinical evaluation of wound healing, pain, shoulder function and range was done. Results were evaluated by the use of Constant and Murley shoulder score based on pain, activities of daily living, range of motion and strength.

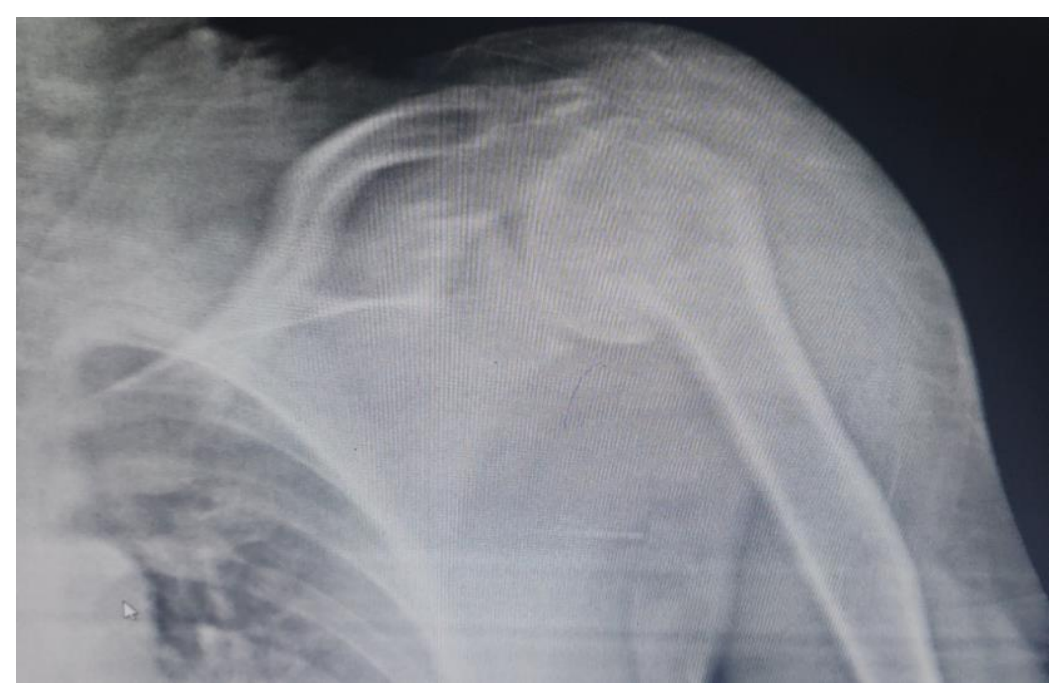

Fig 1: Xray AP view of Left shoulder showing a Neer's 3 part Proximal Humerus fracture in a 62 year old female

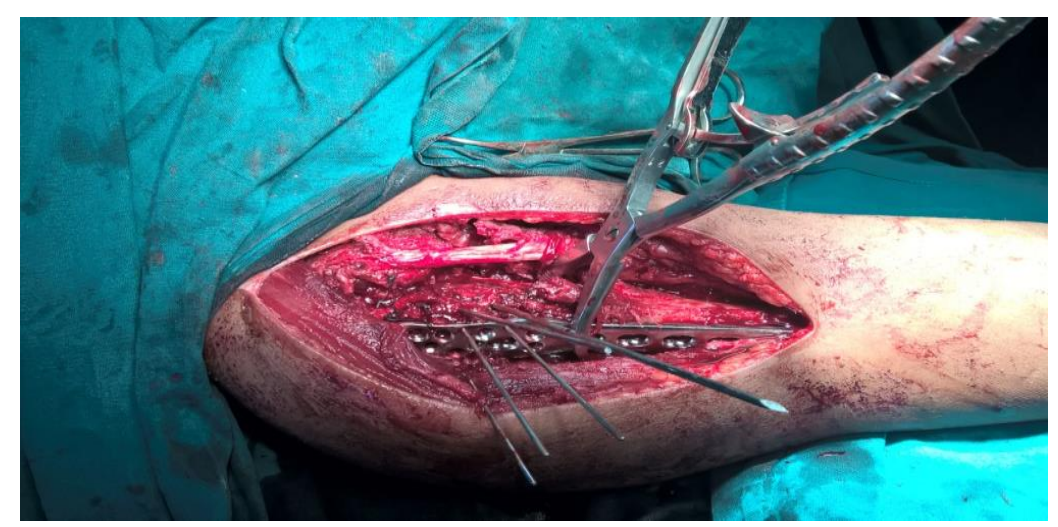

Fig 2: Intraoperative picture showing provisional reduction with $\mathrm{k}$ wires and plate position 


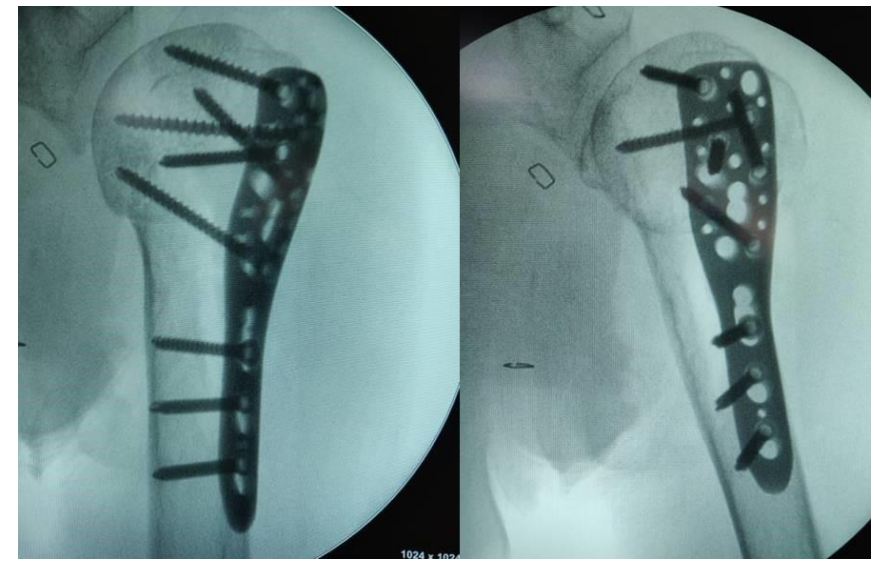

Fig 3: Final intra operative Fluoroscopy images of the patient whose pre operative image was depicted in Figure 1

\section{Results}

A total of 30 patients were included in our study. Age of the study subjects were ranging between 19 and 68. Mean age of the study group was 44.4 with standard deviation of 14.67 . Among 30 subjects $40 \%$ (12) were females rest $60 \%$ (18) were males. $26.7 \%$ (8) encountered fracture due to fall from surface, $53.3 \%$ (16) due to road traffic accident and rest $20 \%$ (6) from seizure. Majority of them had Neers 3 part fracture $56.66 \%$ (17), Neers 4 part fracture $23.33 \%$ (7) followed by Neers 2 part fracture $13.33 \%$ (4). Among those who had Neers 3 part fracture, $64.7 \%$ (11) did not have associated dislocation and rest $35.29 \%$ (6) had either anterior or posterior dislocation. Most of them had fracture of the right limb $66.66 \%$ (20) and rest of them $33.33 \%$ (10) had it in their left limb. Among them 50\% (15) of subjects had associated injuries like injury to facial bone, head injury, contralateral radius fracture, foot injuries, vertebral fracture and fractures of the femur or tibia. Median days of interval between injury and surgery were 6 days with the interval ranging between 1 to 45 days. The mean Constant and Murley score at 6 weeks was 28.2 with standard deviation of 10.34 . Mean score at 3 months was 57.86 with standard deviation of 14.62 with lost followup of 1 subject. Median score at 6 months was 72.5 with the scores ranging between 32 and 92 with $n$ of 25 with lost followup of 5 subjects. At the end of 6 months, 8 subjects had excellent outcome, 11 had good outcome. 3 patients had a moderate outcome and 3 patients had a poor outcome. Hence, the number of patients with a satisfactory outcome were 19 $(76 \%)$.

\section{Complications}

Major adverse outcome were varus malunion 10\% (3) and GT malunion $10 \%$ (3) and 3.3\% (1) had to undergo bone grafting. There were no cases of avascular necrosis, implant breakage, screw perforation, infection or implant breakage.

\section{Discussion}

Of all fractures of long bones, 4-5\% of them are proximal humerus fractures ${ }^{[1]}$. With the increase in geriatric population with osteoporosis, its incidence is increasing. The incidence is also increasing in the younger population due to the increase in incidences of road traffic accidents. Palvanen et al. ${ }^{[60]}$, in their study, determined the trend in the fall related, and osteoporosis related proximal humeral fractures in elderly Finnish population. They predicted that the number of fractures would triple in the next three decades.

Majority (80-85\%) of these fractures can be managed conservatively. The remaining $15-20 \%$ are significantly displaced and require some type of internal fixation. Treatment options for the fixation of proximal humerus fractures include $\mathrm{K}$ wires, T-buttress plate, hemiarthroplasty and semitubular plates. In non-osteoporotic bones, open reduction and internal fixation with non locking plates and screws for proximal Humerus fractures has shown to provide the strongest fixation as shown by Wijgman et al. ${ }^{[7]}$. In their study the mean age group was forty eight years. However, in patients with osteoporosis the bone quality decreases and effectiveness of non locking plates decreases, as the stability of non locking plates depends on friction between plate and bone. Complications such as screw loosening resulting from insufficient purchase leads to high failure rate especially in three part and four part fractures. Kristiansen et al. ${ }^{[8]}$ in their study of 20 patients with proximal Humerus fracture treated with T-buttress plate, found that only nine reported a satisfactory or excellent result and all four part fractures resulted in poor outcomes.

A thorough understanding of the injury and analysis of literature for surgical management is required as these fractures present with difficulty while reducing and fixing displaced proximal humerus fracture or fracture dislocation. Management of the fracture in elderly low demand patient has also been a dilemma ${ }^{[9]}$.

Proximal humerus locking plate was implemented to overcome these complications. It is the most commonly used implant at present for these fractures, as it permits indirect reduction of the articular fragments using image intensifier, thus lowering the possibility of AVN particularly in four part fractures.

However, as the need is for a good reduction, early mobilization and early fracture healing for restoring functionality of the limb, open reduction and internal fixation with locking compression plate is a preferable mode of treatment.

This study was carried from January 2019 to May 2019 in the department of Orthopedics, Sanjay Gandhi Institute of Trauma and Orthopaedics, Bengaluru. A total of 30 patients were included out of which only 25 patients followed up entirely and were included for the final statistical analysis.

The results of our study are compared with the various prospective studies conducted in other parts of the world.

The average age in our series of 30 patients, ranging between 19 to 68 years was 44.4 years which is comparable to that of other studies. The average age incidence in Gerber et al. ${ }^{[10]}$ was 44.9 years. Comparative studies showed that the most common age group was the middle aged patient and this could be explained by the common observation that middle aged are more active and the working group of a population.

Regarding sex incidence, study of literature reveals predominance of proximal humeral fractures in females in an elderly age group. Studies also reveal that male to female ratio being 1:0.8. In our series the male to female ratio is $3: 2$. The reason for high incidence of males in our series is because majority of the cases, 13 out 30 were under the age of $50 y e a r s$. The fractures of proximal humerus have a bimodal presentation with younger patients; predominantly males are prone for high velocity injuries. This is due to outdoor work related activities. In older patients, fractures are osteoporosis related and most often seen in females. The mode of injury observed in our series was road traffic accidents accounting for $53.3 \%, 26.7 \%$ patients having a slip and fall and $20 \%$ occurred after seizures. These observations were found to be consistent with the studies in literature which revealed $45 \%$ road traffic accidents and $50 \%$ history of slip and fall ${ }^{[7]}$. The 
incidence of RTA is more in our study because of many patients being young (less than 45 years). The most common mode of injury in young patients is RTA and in elderly it is domestic fall, which is consistent with literature. Six patients had fractures following seizure episodes.

Our study series revealed $13.33 \%$ two part fractures, $56.66 \%$ three part fractures, $23.33 \%$ four part fracture and of these, $35.29 \%$ were fracture dislocations. Felix Brunner et al. [11] showed similar incidences, except for higher incidence of fracture dislocation. This is because of limited sample size and majority had RTA as the cause of trauma.

The average Constant and Murley score in our study was found to be 72.5.A systematic review of 12 studies done by Robert et al. ${ }^{[12]}$ had a mean score of 74 . Greiner et al. ${ }^{[13]}$ and Sharafeldin et al. ${ }^{[14]}$ had a mean Constant score comparable to our study that is 66 and 65 respectively.

Greiner et al. ${ }^{[13]}$ observed a Constant score of $66.2 \pm 15.4$ at the mean follow up of 45 months. We observed a constant score of 72.5 at the mean follow up six months. They also noted that the constant score improved during the 6 months follow up ( constant score $71.6 \pm 18.3$ ) \% to 12 months follow up $(77.7 \pm 17.8) \%$. However they did not notice significant improvement at 45 months follow up. With longer duration of follow up, incidence of AVN was more, i.e. 4 cases at 12 months and 9 cases by final follow up.

Sharafeldin et al. ${ }^{[14]}$ and Robert et al. ${ }^{[12]}$ noted that the constant scores significantly worsened as the severity of the fracture increased. However in our study we found that two part fracture had the worst functional outcome because of the preexisting rotator cuff injury. Barring this exception of two part fracture patient, the Constant scores worsen as the severity increases. This is statistically significant (p value<0.05)

The ideal plate position as described by $\mathrm{AO}{ }^{[15]}$ is $5-8 \mathrm{~mm}$ distal to the tip of the greater tuberosity and 2-4 $\mathrm{mm}$ posterior to the bicipital groove. We intended to find out the functional outcome with regards to plate placement.70\% of the patients had a desired optimum plate placement and the results at the end of six months show that $85 \%$ of these patients had good to excellent outcome which was statistically significant ( $p$ value $=0.0210$. Siwach et al. ${ }^{[16]}$ followed the principles of definitive fixation, with plate positioned lateral to the bicipital tendon and at least $1 \mathrm{~cm}$ distal to the top of greater tuberosity. However they did not compare the functional outcome with optimal plate placement.

Hertel et al. ${ }^{[17]}$ compared the proximal Humerus to a cracked eggshell. The stability of the construction relies on the restoration of the circular integrity of the egg cup i.e. the tuberosity. The head is allowed to sit comfortably on the corticocancellous rim provided by the reduced tuberosities. Accepting less than anatomic reduction of tuberosities greatly reduces the intrinsic stability of the construction. Aggarwal et al. ${ }^{[18]}$ and Siwach et al. ${ }^{[16]}$ did not directly correlate the greater tuberosity reduction and its relation to functional outcome. Our study revealed positive correlation between greater tuberosity reduction and final outcome. $85 \%$ of the patients with greater tuberosity reduction had good to excellent outcomes at the end of six months which was statistically significant ( $\mathrm{p}$ value $=0.021$ ).

Proximal humerus fractures are known to have complications like varus malunion, AVN, Screw perforation, subacromial impingement, infection, non union and axillary nerve palsy but we noticed only malunions as complications in six patients. Among these three patients had varus malunion and three patients had greater tuberosity malunion.
Patients without any complications attained statistically significant ( $\mathrm{p}$ value $=0.0361$ ) functional outcome as compared to patients with complications. Aggarwal et al. ${ }^{[18]}$ in their study encountered complication like screw back out, screw perforation, AVN humeral head, subacromial impingement, axillary nerve palsy and wound infections. Majority of their complications were attributed to the fact that they had occurred during their initial experience and as the experience and learning curve had increased the complication rate reduced. Complications were avoided by anatomic reduction at the time of surgery, provisionally fixing with $\mathrm{K}$ wires and getting confirmatory radiographs though the arc of motion. They also avoided screw perforation by putting smaller sized screws when the measured length fell between two screw sizes. They had developed wound infection due to poor soft tissue handling and excessive skin flap in their initial period. We had avoided raising excessive flaps, used shorter sized screws and intra operative imaging. Hence we had no incidence of infection, screw perforation and plate impingement. In our three patients who had greater tuberosity malunion two patients had unsatisfactory results. The patients who had a varus malunion had obtained good to moderate results. This correlated with the results of Greiner et al. ${ }^{[13]}$ who noted that the presence of varus angulation did not result in significant differences in the mean constant scores at six months, twelve months and final follow up.

All the malunions which we have noticed were due to primary varus fixation and inadequate greater tuberosity reductions at the time of surgery. Siwach et al. ${ }^{[16]}$ noted $8 \%$ of malunions which was also primary varus fixation at the time of surgery. They however did not consider and discuss regarding the greater tuberosity malunion. Since we have considered both varus and greater tuberosity malunion, we have noted a higher incidence of malunions. They also did not include four part fractures in their study population whereas we had $23.33 \%$ four part fractures.

According to Sproul et al. ${ }^{[12]}$ screw perforation could be due to primary, an unrecognized perforation or secondary, occurring as a result of collapse of the humeral head due to AVN or varus malunion. However we avoided primary screw perforation by intraoperative imaging through complete arc of motion and also by using shorter screws at the time of surgery.

Avascular necrosis of the humeral head was known to occur upto five years after surgery. However our study was limited to six months follow up and hence we didn't notice AVN and secondary screw perforation.

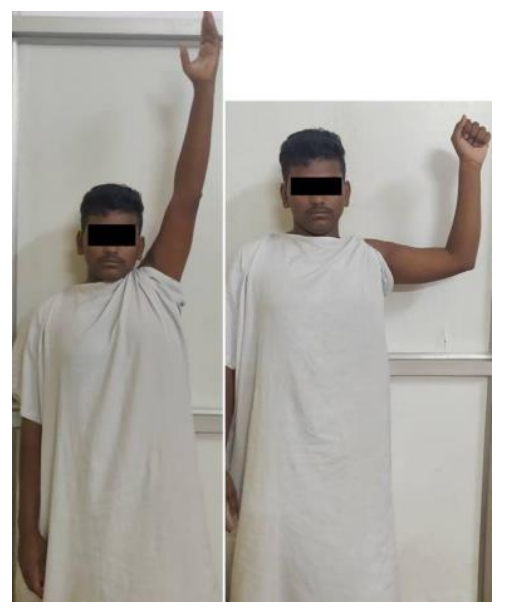

Fig 4: Over head abduction and External rotation of a patient with excellent outcome following union 


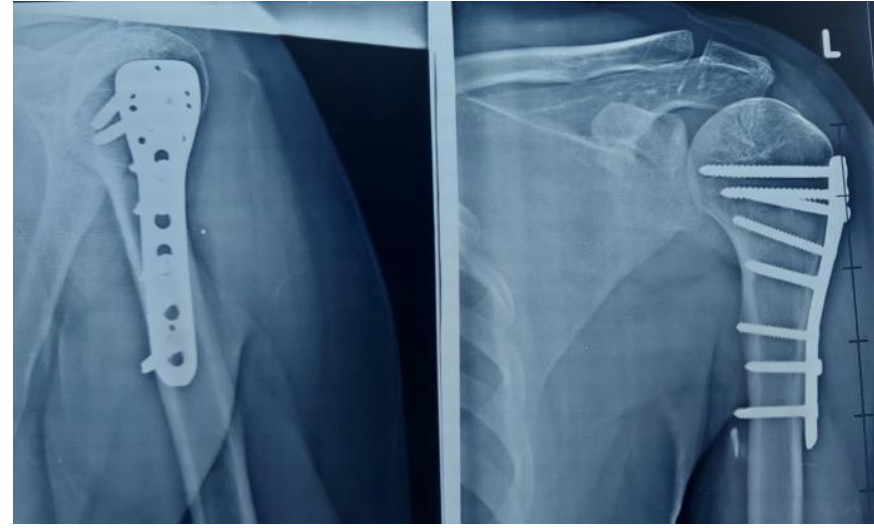

Fig 5: Xray images of the patient in Fig 4 following union

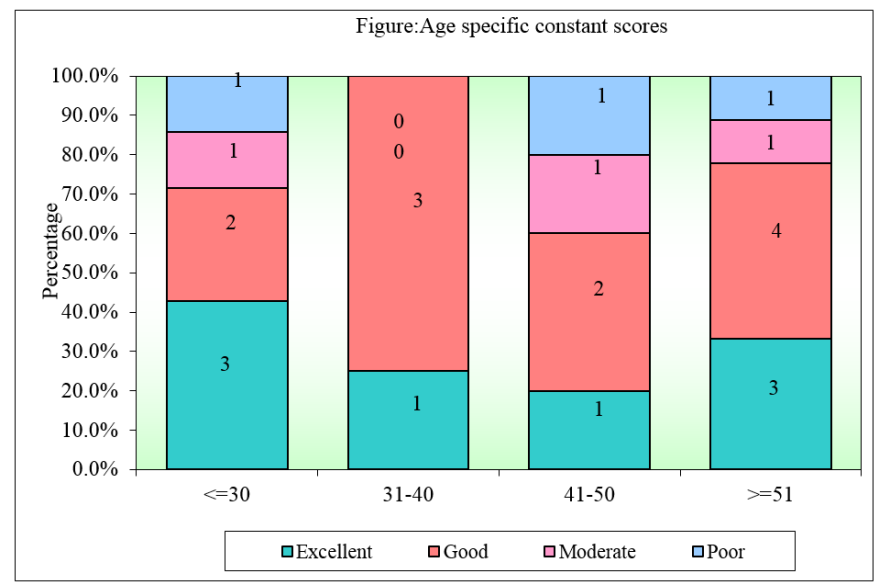

Fig 6: Age Specific Constant and Murley Scores

\section{Limitation of Study}

- Limited Sample size.

- It's not a case controlled study.

- Study period was limited to only six months. Complications like AVN and screw perforation can occur even upto five years after surgery.

- $\quad$ Surgery was performed by multiple surgeons with varied experience and technique.

- Physiotherapy in the post op period was not followed by some patients due to low educational status and difficulty in commuting from far off places for follow up.

- Proximal humerus locking plates used in this study was not from the same company for all the patients.

\section{Conclusion}

Our study concludes that Proximal humerus fractures in young are mainly due to high energy injuries like RTA whereas in elderly, fractures are mainly due to low energy slip and fall. High energy injuries and seizure disorders resulted in more communitted three part and four part fractures. Low energy injuries resulted in less communitted fractures. At six months follow up, the functional outcome assessed by Constant and Murley score revealed a good to excellent outcome in $76 \%$ of patients. Barring the one patient with rotator cuff injury, the functional outcome decreases with increasing severity of the proximal humerus fractures ( $p$ value $=0.0210$ ). Average union time for proximal humerus fractures treated with proximal humerus locking plates is 17.06 weeks. Proper placement of the plate, $5-8 \mathrm{~mm}$ from the tip of the greater tuberosity and greater tuberosity reduction is the key to better functional outcome. ( $p$ value $=0.0210)$. Proximal humerus locking plates gives stable fixation in proximal humerus fractures and prevents secondary collapse. Intra operative imaging through the arc of motion and also selecting shorter sized screws for the head avoids primary screw perforation.

\section{References}

1. Court Brown CM, Caesar B. Epidemiology of adult fractures: A review Injury 2006; 37:691-697.

2. Terry Canale's Campbell's Operative Orthopaedics, Vol3: $9^{\text {th }}$ edition, 1998 Mosby Publishers, USA, 2286-2296.

3. Steven H. Rose Joseph Melton Bernard.F.Morrey et al. Epidemiological features of humeral fractures Clin. Orthop. 1982; 168:24-30.

4. RL Sahu. Philos Locking plates in proximal Humerus fractures literature review. The Internet Journal of Health. 2010, 11.

5. Bigliani LU, Flatow EL, Pollock RG. Fractures of the proximal Humerus In: Rockwood CA, Green DP, Bucholz RW, Heckman JD, eds. Fractures In adults. Philadelphia, etc: Lippincott-Raven, 1996, 1055-107.

6. Palvanen M, Kannus P, Niemi S, Parkkari J: Update in the epidemiology of proximal humeral fractures. ClinOrthop Related Res. 2006; 442:87-92.

7. Wijgman AJ, Roolker W, Patt TW et al. Open reduction and internal fixation of three and four part fractures of proximal Humerus. Scientific Article November01, 2002.

8. Kristiansen B, Christensen SW. Plate fixation of proximal humeral fractures. Acta Orthop Scand 1986; 68 A: 1410-1414.

9. Jan-Magnus Björkenheim, JarkkoPajarinen et al, "Internal fixation of proximal humeral fractures with a locking compression plate" ActaOrthopScand. 2004; 75(6):741-745.

10. Gerber C, Worner CM, Vienne P. "Internal fixation of complex fractures of the proximal Humerus". J Bone Joint Surg (Br). 2004; 86(60):848-855.

11. Felix Brunner, Christoph Sommer, Christian Bahrs et al Open Reduction and Internal Fixation of Proximal Humerus Fractures Using a Proximal Humeral Locked Plate: A Prospective Multicenter Analysis, J Orthop Trauma.

12. Robert CS, Jaicharan Iyengar J, ZlatkoDevcic, Brian Feeley T. A systematic review of locking plate fixation of proximal humerus fractures. Injury, Int. J Care Injured. 2011; 42:408-41.

13. Greiner S, Kaab MJ, Haas NP, Bail HJ. Humeral head necrosis rate at mid-term follow-up after open reduction and angular stable plate fixation for proximal humeral fractures. Injury, Int. J. Care Injured. 2009; 40:186-19.

14. Sharafeldin KN, Quinlan JF, Corrigan J et al. Functional follow-up of locking plate fixation of fractures of the proximal humerus. Eur J OrthopSurg Trauma. 2008; 18:87-92.

15. Kocher T. Beitragezur Kenntniseinigerpraktischwichtige Frakturenformen. Basel: Karl Sallman Verlag, 1896.

16. Ramchander Siwach Roop Singh, Rajesh Kumar Rohilla et al. "Internal fixation of proximal Humerus fracture by locking proximal Humerus plate in elderly osteoporotic" J Orthopaed Traumatol. 2008; 9:149-153.

17. Ralph Hertel. Fractures of the proximal humerus in osteoporotic bone. Osteoporosis Int. 2005; 16:S65-S72.

18. Sameer Aggarwal et al. "Displaced proximal humeral fractures: an Indian experience with locking plate". Journal of Orthopaedic surgery and research. 2010; 5:60. 